\title{
La jeune génération doit tout réinventer
}

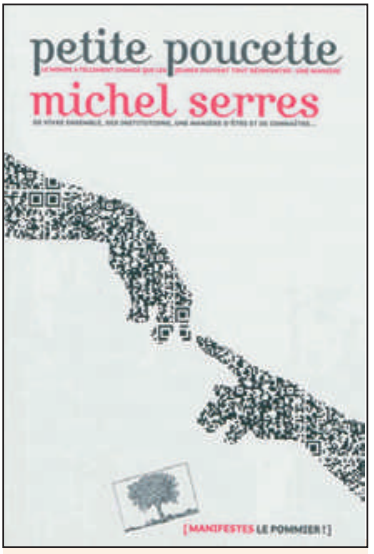

Michel Serres Petite Poucette

Paris: Ed. Le Pommier, 2012 84 pages. $16 \mathrm{CHF}$ ISBN 978-2-7465-0605-3
Michel Serres est ce philosophe et historien des sciences français, qui a commencé par être officier de marine avant de se tourner vers l'épistémologie puis l'enseignement, en particulier depuis trente ans à l'Université de Stanford. Il pose un regard érudit, perspicace, incisif mais aussi empathique sur notre monde et son évolution. Avec des allures de feu d'artifice intellectuel. La liste de ses ouvrages est impressionnante (plus de soixante). Il fut un temps où mon espoir était de lire la plupart de ce qu'il écrivait, j'ai massivement échoué. Un des derniers, court, me donne l'occasion de reprendre contact: Petite Poucette. Cette héroïne représente les jeunes d'aujourd'hui, elle tient son nom du fait qu'ils travaillent beaucoup, constamment, avec leurs deux pouces, sur leur tablette ou leur I-phone.

Premières lignes: Ce nouvel écolier n'a jamais vu «veau, vache, cochon ni couvée» (élément de phrase du fabuliste La Fontaine - je pense pour ma part souvent au fait que plus nombreux chaque jour sont les enfants qui ne savent guère d'où, dans la nature, vient le lait, s'il est normal que les vaches n'aient pas de cornes, avec quoi on fait du pain et si c'est sous la terre ou en l'air qu'on trouve des oignons, des arachides, des pêches ou des ananas pour les mettre en conserve). «En 1900, la majorité des humains sur la planète travaillaient au labour ou à la pâture; en l'histoire (encore que la qualité des informations ne soit pas garantie). La mémoire, ce qu'a mémorisé chacun, critère majeur de qualification scolaire et académique il y a encore une génération, perd beaucoup de son importance. «Les jeunes sont formatés par des médias, diffusés par des adultes, qui ont méticuleusement détruit leur faculté d'attention en réduisant la durée des images à sept secondes...»; «Nous avons transformé notre société du spectacle [du multimédia] en une société pédagogique dont la concurrence écrasante, vaniteusement inculte, éclipse l'école et l'université».

Fin de l'érudition au sens classique? «Si elle a consulté un bon site sur la Toile, Petite Poucette peut en savoir autant ou plus sur le sujet traité, la décision à prendre, le soin de soi, qu'un maître, un directeur, un grand patron, un élu. Combien d'oncologues avouent avoir plus appris sur les blogs des femmes atteintes d'un cancer du sein qu'à la faculté? Les spécialistes d'histoire naturelle ne peuvent plus ignorer ce que disent, en ligne, les fermiers australiens sur les mœurs des scorpions ou les guides des parcs pyrénéens sur le déplacement des isards [chamois]. Le partage symétrise l'enseignement; le retournement du vieil iceberg [dont la pointe était le professeur] favorise une circulation à double entente». Ce qui appelle évidemment des changements de paradigme,

\section{La Petite Poucette représente les jeunes d'aujourd'hui, elle tient son nom du fait qu'ils travaillent beaucoup avec leurs deux pouces, sur leur tablette ou leur I-phone.}

2011, la France ne compte plus que un pour cent de paysans. Sans doute faut-il voir là une des plus fortes ruptures de l'histoire (...) Son espérance de vie va vers quatre-vingt ans. A leur mariage ses arrière-grands-parents s'étaient juré fidélité pour une décennie à peine». Rappelant aussi que, aujourd'hui, le multiculturalisme est de règle.

«Quelle litterature, quelle histoire comprendront-ils, heureux [Petite Poucette et ses contemporains], sans avoir vécu la rusticité, les bêtes domestiques, dix conflits, cimetières, blessés et affamés, sans avoir expérimenté dans la souffrance l'urgence vitale d'une morale?». Qui peut répondre?

Nous peinons tous à imaginer l'impact de la disponibilité immédiate, par les moyens d'aujourd'hui de (presque) tout ce qui est connu et l'a été à cours de dans l'enseignement, dans la pratique médicale, ailleurs - changements dans les accents mis sur telle ou telle dimension du rôle du professionnel (savoir, compétences, qualités relationnelles et de transmission).

On entend de plus en plus de préoccupations et de critiques sur la difficulté d'institutions prestigieuses (par exemple et parmi d'autres ce qu'on appelle en France les Grandes Ecoles, portes d'entrée aux emplois élevés de l'Etat et de l'économie) à s'adapter - à cause de rigidités et traditions - à la société actuelle mobile, moins hiérarchique, plus flexible. Serres a une forte métaphore: «Je vois nos institutions luire d'un éclat semblable à celui des constellations dont les astronomes nous apprennent qu'elles sont mortes depuis longtemps déjà». 


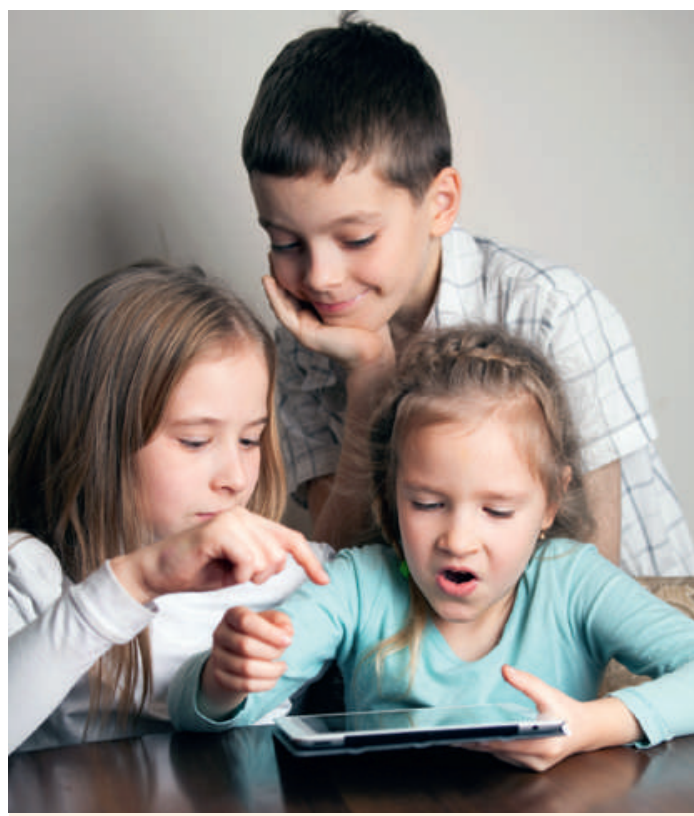

Ont-ils vu veau, vache, cochon ou couvée?

A propos de l'accumulation de données personnelles par des intervenants divers et multiples, thème devenu banal mais pas moins inquiétant: «Petite Poucette - individu, client, citoyen - laissera-t-elle l'Etat, les banques, les grands magasins... s'approprier ses données propres, d'autant qu'elles deviennent une source de richesse. Il peut en résulter un regroupement des partages socio-politiques par l'avènement d'un cinquième pouvoir, celui des données».

Autre préoccupation lancinante, que personne n'entend aborder de front parmi les décideurs de la politique ou de l'économie: nos sociétés peuventelles continuer à affirmer de manière crédible l'objectif d'assurer l'emploi de (presque) tous? Serres: «La productivité, augmentant verticalement, et la crois-

\section{Un regard érudit, perspicace, incisif mais aussi}

\section{empathique sur notre monde et son évolution.}

\footnotetext{
* Est dit algorithmique un processus systématique de résolution décrivant les étapes vers le résultat; un algorithme est une suite d'instructions permettant de donner la réponse à un problème.
}

sance démographique raréfient de plus en plus le travail; une aristocratie en bénéficiera-t-elle seule?». Je sais que les nouvelles technologies, parmi d'autres, sont créatrices d'emplois. Mais, rien que sur un point: est-il imaginable que tout un chacun ait les compétences qui seront alors requises? Alors que, jusqu'au milieu du XX $\mathrm{XX}^{\mathrm{e}}$ siècle, il y avait quantité de travaux qui ne nécessitaient que des muscles et des articulations en relatif bon état.

Sur le rôle, pourtant plus nécessaire que jamais du politique, Serres relève qu'il n'y a plus des conducteurs et des passagers mais que tout le monde veut être conducteur (je trouve aussi que nos sys- tèmes démocratiques vivent des dérives selon quoi tout le monde serait aussi compétent, pour décider de tout, qu'un autre, ce dernier fût-il conseiller fédéral). «Il n'y a plus de puissants dans l'arène politique, désormais occupée par des décidés. Fin de l'ère du décideur» (j'ai trouvé un rien énigmatique).

Il fait l'éloge des réseaux qui remplacent les anciens groupes de référence: «Agonisent les vieilles appartenances: fraternités d'armes, paroisses, partis, syndicats, familles en recomposition; restent les groupes de pression, obstacles honteux à la démocratie» (bonne chose que quelqu'un le dise clairement). Cela étant, il faut se souvenir que: «Sanguinaires, ces appartenances exigeaient que chacun fît sacrifice de sa vie: martyrs suppliciés, femmes lapidées, hérétiques brûlés vifs, prétendues sorcières immolées, soldats inconnus alignés par milliers, camps d'extermination et goulags...».

«A ces appartenances, à ces faux dieux mangeurs de victimes infinies, je préfère, dit Serres revenant à aujourd'hui, notre virtuel immanent, qui comme l'Europe ne demande la mort de personne. Ne plus construire un collectif sur le massacre d'un autre et le sien propre». Utile, au sein des turbulences que connaît l'Union européenne, de rappeler qu'elle a assuré durant plus d'un demi-siècle déjà une convivialité inconnue auparavant.

Des remarques particulièrement stimulantes sur la (nouvelle) importance de l'algorithmique* et du procédural: «Observez Petite Poucette manipuler un téléphone portable: elle déploie sans hésitation un champ cognitif qu'une part de la culture antérieure, celle des sciences et des lettres, a longtemps laissé en jachère, que l'on peut nommer procédural. Ces procédures sont en passe de concurrencer l'abstrait, la géométrie par exemple, et pénètrent les savoirs et les techniques (...) Désormais, l'atterrissage d'aéronefs sur des pistes fréquentées, une longue opération du rein ou du cœur, la fusion de deux sociétés industrielles, le dessin d'une puce, exigent des conduites différentes de la déduction du géomètre ou de l'induction expérimentale». Pourtant aussi philosophe lui-même, Serres voit cette discipline en grave décalage: «Le collectif, le technologique, l'organisationnel se soumettent plus, aujourd'hui, à ce cognitif procédural qu'aux abstractions déclaratives que la philosophie consacre depuis deux millénaires. Simplement analytique, elle ne voit pas ce cognitif s'instaurer. Elle rate notre temps».

J'ai entendu des commentateurs faire à ce petit livre la critique que l'auteur y décrit des préoccupations déjà largement connues et discutées par d'autres, intellectuels ou politiques. C'est en partie vrai. Mais il reste à mon sens un maître à penser dynamique (et attachant, avec son accent du Sud-Ouest qui ne saurait paraître à l'écrit!). En fait, il refuserait probablement le terme de maître à penser et préférerait être vu comme quelqu'un qui pointe les problèmes et accouche des questions (comme Socrate), cherchant à stimuler ceux qui le lisent à réfléchir et à agir. 